PROCEEDINGS OF THE

AMERICAN MATHEMATICAL SOCIETY

Volume 136, Number 9, September 2008, Pages 3293-3300

S 0002-9939(08)09487-2

Article electronically published on April 17, 2008

\title{
ON THE ISOTROPY CONSTANT OF RANDOM CONVEX SETS
}

\author{
DAVID ALONSO-GUTIÉRREZ
}

(Communicated by N. Tomczak-Jaegermann)

\begin{abstract}
Let $K$ be the symmetric convex hull of $m$ independent random vectors uniformly distributed on the unit sphere of $\mathbb{R}^{n}$. We prove that, for every $\delta>0$, the isotropy constant of $K$ is bounded by a constant $c(\delta)$ with high probability, provided that $m \geq(1+\delta) n$.
\end{abstract}

\section{INTRODUCTION AND NOTATION}

A convex body $K \subset \mathbb{R}^{n}$ is a compact convex set with non-empty interior. A convex body is said to be in isotropic position if it has volume 1 and satisfies the following two conditions:

- $\int_{K} x d x=0$ (center of mass at 0 ),

- $\int_{K}\langle x, \theta\rangle^{2} d x=L_{K}^{2} \quad \forall \theta \in S^{n-1}$,

where $L_{K}$ is a constant independent of $\theta$, which is called the isotropy constant of $K$. Here $\langle\cdot, \cdot\rangle$ denotes the standard scalar product in $\mathbb{R}^{n}$.

It is well known that for every convex body $K \subset \mathbb{R}^{n}$ there exists an affine map $T$ such that $T K$ is isotropic. Furthermore, $K$ and $T K$ are both isotropic if and only if $T$ is an orthogonal transformation. In this case the isotropy constant of both $K$ and $T K$ are the same. Hence, we can define the isotropy constant for every convex body. It satisfies the following equation:

$$
n L_{K}^{2}=\min \left\{\frac{1}{|T K|^{1+\frac{2}{n}}} \int_{a+T K}|x|^{2} d x \mid a \in \mathbb{R}^{n}, T \in G L(n)\right\},
$$

where $|\cdot|$ denotes both the volume of a set in $\mathbb{R}^{n}$ and the Euclidean norm of a vector. We refer the reader to 9 for these and other well-known facts concerning isotropic convex bodies.

It is conjectured that there exists an absolute constant $C$ such that for every convex body $K, L_{K} \leq C$. This conjecture has been verified for several classes of convex bodies such as unconditional bodies [9, zonoids [1] and others (e.g., 7]). However, the best known general upper bound is $L_{K} \leq c n^{\frac{1}{4}}$, [5], which improves the earlier estimate $L_{K} \leq c n^{\frac{1}{4}} \log n$ given by Bourgain (see [2]). In a recent paper 6], Klartag and Kozma proved that with high probability the conjecture is true for the convex hull of independent Gaussian vectors and suggested that with the same techniques it should be possible to obtain an analogous result for the convex

Received by the editors July 10, 2007.

2000 Mathematics Subject Classification. Primary 52A20.

The author was supported by an FPU Scholarship from MEC (Spain), MCYT Grants (Spain) MTM2007-61446, DGA E-64 and by Marie Curie RTN CT-2004-511953.

(C)2008 American Mathematical Society 3293

Reverts to public domain 28 years from publication 
hull of independent points uniformly distributed on the sphere (in this case the coordinates are not independent). In this paper we are going to prove this result. To be precise, we are going to prove the following theorem:

Theorem 1.1. For every $\delta>0$, there exist constants $c(\delta), c_{1}$ and $c_{2}$ such that if $m>(1+\delta) n,\left\{P_{i}\right\}_{i=1}^{m}$ are independent random vectors on $S^{n-1}$ and $K=$ $\operatorname{conv}\left\{ \pm P_{1}, \ldots, \pm P_{m}\right\}$, then

$$
\mathbb{P}\left\{L_{K} \leq c(\delta)\right\} \geq 1-c_{1} e^{-c_{2} n \min \left\{1, \log \frac{m}{n}\right\}} .
$$

In this paper we will denote by $\sigma$ the uniform probability measure on the sphere $S^{n-1}$ and by $\omega_{n}$ the volume of the Euclidean ball. Hence, $\Delta^{n-1}=\operatorname{conv}\left\{e_{1}, \ldots, e_{n}\right\}$, where $\left\{e_{i}\right\}$ is the standard unit vector basis of $\mathbb{R}^{n}$. The letters $C, c, c_{1}, c_{2}, \ldots$ will always denote absolute constants whose value may change from line to line.

\section{Some Previous RESUlts}

In this section we are going to recall some known facts that we are going to use. We begin with

Definition 2.1. We write $L_{\psi_{2}}$ for the space of real-valued measurable functions on $S^{n-1}$ such that $\int_{S^{n-1}} e^{\frac{|f|^{2}}{\lambda^{2}}} d \sigma<\infty$ for some $\lambda>0$, and we set

$$
\|f\|_{L_{\psi_{2}}}=\inf \left\{\lambda>0: \int_{S^{n-1}} e^{\frac{|f|^{2}}{\lambda^{2}}} d \sigma<2\right\} .
$$

Bernstein's inequality gives a bound for the probability that the absolute value of the sum of $N$ independent random variables with mean 0 is bigger than $\epsilon N$ for certain values of $\epsilon$. There are several versions of this inequality, depending on the space to which the random variables belong. We are going to use the following version, whose proof can be found in $[3]$.

Theorem 2.1 (Bernstein's inequality). Assume $\left\{g_{j}\right\}_{j=1}^{N} \subset L_{\psi_{2}}$ are independent random variables with zero mean such that $\left\|g_{j}\right\|_{L_{\psi_{2}}} \leq A$ for all $j$ and some constant A. Then, for all $\epsilon>0$,

$$
\mathbb{P}\left\{\left|\sum_{j=1}^{N} g_{j}\right|>\epsilon N\right\} \leq 2 e^{-\frac{\epsilon^{2} N}{8 A^{2}}} .
$$

We will apply this inequality to the independent identically distributed random variables $\sqrt{n}\langle P, \theta\rangle$, where $P$ is a point distributed uniformly in the unit sphere. To see that these random variables are in $L_{\psi_{2}}$ we need the following lemma:

Lemma 2.1. For all $q \geq 1$ and for all $\theta \in S^{n-1}$

$$
\int_{S^{n-1}}|\langle u, \theta\rangle|^{q} d \sigma(u)=\frac{2 \Gamma\left(\frac{1+q}{2}\right) \Gamma\left(1+\frac{n}{2}\right)}{\sqrt{\pi} n \Gamma\left(\frac{n+q}{2}\right)} .
$$

Proof. Changing to polar coordinates in the following integral, we have that

$$
\begin{aligned}
\int_{\mathbb{R}^{n}}|\langle x, \theta\rangle|^{q} \frac{e^{-\frac{|x|^{2}}{2}}}{(\sqrt{2 \pi})^{n}} d x & =\frac{n \omega_{n}}{(\sqrt{2 \pi})^{n}} \int_{0}^{\infty} r^{n+q-1} e^{-\frac{r^{2}}{2}} d r \int_{S^{n-1}}|\langle u, \theta\rangle|^{q} d \sigma(u) \\
& =\frac{2^{\frac{q}{2}} n \Gamma\left(\frac{n+q}{2}\right)}{2 \Gamma\left(1+\frac{n}{2}\right)} \int_{S^{n-1}}|\langle u, \theta\rangle|^{q} d \sigma(u) .
\end{aligned}
$$


On the other hand, since

$$
\begin{aligned}
\int_{\mathbb{R}^{n}}|\langle x, \theta\rangle|^{q} \frac{e^{-\frac{|x|^{2}}{2}}}{(\sqrt{2 \pi})^{n}} d x & =\int_{-\infty}^{\infty}|x|^{q} \frac{e^{-\frac{x^{2}}{2}}}{\sqrt{2 \pi}} d x=\frac{2}{\sqrt{2 \pi}} \int_{0}^{\infty} x^{q} e^{-\frac{x^{2}}{2}} d x \\
& =\frac{2^{\frac{q}{2}} \Gamma\left(\frac{1+q}{2}\right)}{\sqrt{\pi}}
\end{aligned}
$$

we obtain the result.

Remark. By Stirling's formula we have that there exist absolute constants $c_{1}$ and $c_{2}$ such that for all $q \geq 1$ and for all $\theta \in S^{n-1}$ :

$$
c_{1} \sqrt{\frac{q}{q+n}} \leq\left(\int_{S^{n-1}}|\langle u, \theta\rangle|^{q} d \sigma(u)\right)^{\frac{1}{q}} \leq c_{2} \sqrt{\frac{q}{q+n}} .
$$

Corollary 2.1. There exists a constant $A>0$ such that if $\theta$ is a unit vector, then the functional $\sqrt{n}\langle P, \theta\rangle$ satisfies $\|\sqrt{n}\langle\cdot, \theta\rangle\|_{L_{\psi_{2}}} \leq A$.

Proof. Let $\theta$ be a unit vector. Then

$$
\begin{aligned}
\int_{S^{n-1}} e^{\frac{n|\langle P, \theta\rangle|^{2}}{A^{2}}} d \sigma(P) & =\sum_{q=0}^{\infty} \int_{S^{n-1}} \frac{n^{q}|\langle P, \theta\rangle|^{2 q}}{A^{2 q} q !} d \sigma(P) \\
& \leq 1+\sum_{q=1}^{\infty} \frac{C^{2 q}(2 q)^{q}}{A^{2 q} q !} \\
& \leq 1+C^{\prime} \sum_{q=1}^{\infty}\left(\frac{2 C^{2} e}{A^{2}}\right)^{q} \frac{1}{\sqrt{2 \pi q}} \\
& \leq 1+C^{\prime}\left(\frac{1}{1-\frac{2 C^{2} e}{A^{2}}}-1\right) \leq 2
\end{aligned}
$$

if $A>0$ is chosen large enough.

\section{Symmetric CONVEX HULL OF RANDOM POINTS ON THE SPHERE}

In this section we are going to prove Theorem 1.1

As it was said in the introduction, the isotropy constant of a convex body $K \subset \mathbb{R}^{n}$ satisfies the following equation:

$$
n L_{K}^{2}=\min \left\{\frac{1}{|T K|^{1+\frac{2}{n}}} \int_{a+T K}|x|^{2} d x \mid a \in \mathbb{R}^{n}, T \in G L(n)\right\},
$$

so, in particular

$$
n L_{K}^{2} \leq \frac{1}{|K|^{\frac{2}{n}}} \frac{1}{|K|} \int_{K}|x|^{2} d x .
$$

We will prove our result in two steps: we will give a lower bound for $|K|^{\frac{1}{n}}$ and an upper bound for $\frac{1}{|K|} \int_{K}|x|^{2} d x$ which hold with high probability, and this will imply our statement.

The following lemma is similar to well-known facts about Gaussian polytopes (cf., e.g., [4, 8]). The result is likely known to specialists; as we couldn't find this particular formulation in the literature, we have included the proof. 
Lemma 3.1. For every $\delta>0$, there exists a constant $c(\delta)$ such that if $(1+\delta) n<m<n e^{\frac{n}{2}},\left\{P_{i}\right\}_{i=1}^{m}$ are independent random vectors on $S^{n-1}$, and $K=\operatorname{conv}\left\{ \pm P_{1}, \ldots, \pm P_{m}\right\}$, then

$$
|K|^{\frac{1}{n}} \geq c(\delta) \frac{\sqrt{\log \frac{m}{n}}}{n}
$$

with probability greater than $1-e^{-n}$.

Proof. First observe that, with probability 1, the facets of $K$ are simplices. Let $\alpha \in(0,1)$. If $\alpha B_{2}^{n} \nsubseteq \nsubseteq K$, then there exists a facet of $K$ which lies in a hyperplane orthogonal to some vector $\theta \in S^{n-1}$ such that $\left|\left\langle P_{i}, \theta\right\rangle\right| \leq \alpha$ for all $i$. Let us denote this facet $\operatorname{conv}\left\{Q_{1}, \ldots, Q_{n}\right\}$ with $Q_{j} \in\left\{ \pm P_{1}, \ldots, \pm P_{m}\right\}$ and with $Q_{i} \neq \pm Q_{j}$. It follows that

$$
\mathbb{P}\left\{\alpha B_{2}^{n} \not \subseteq K\right\} \leq\left(\begin{array}{c}
2 m \\
n
\end{array}\right) \mathbb{P}\left\{P \in S^{n-1}:|\langle P, \theta\rangle| \leq \alpha\right\}^{m-n} .
$$

If $\frac{c}{\sqrt{n}} \leq \alpha \leq \frac{1}{4}$, then

$$
\begin{aligned}
\mathbb{P}\left\{P \in S^{n-1}:|\langle P, \theta\rangle|>\alpha\right\} & =2 \frac{(n-1) \omega_{n-1}}{n \omega_{n}} \int_{\alpha}^{1}\left(1-x^{2}\right)^{\frac{n-3}{2}} d x \\
& \geq 2 \frac{(n-1) \omega_{n-1}}{n \omega_{n}} \int_{\alpha}^{2 \alpha}\left(1-x^{2}\right)^{\frac{n-3}{2}} d x \\
& \geq 2 \frac{(n-1) \omega_{n-1}}{n \omega_{n}} \alpha\left(1-4 \alpha^{2}\right)^{\frac{n-3}{2}} \\
& \geq c^{\prime}\left(1-4 \alpha^{2}\right)^{\frac{n-3}{2}} \\
& =c^{\prime} e^{\frac{n-3}{2} \log \left(1-4 \alpha^{2}\right)} \\
& \geq c^{\prime} e^{-4 \alpha^{2} n} .
\end{aligned}
$$

So we have

$$
\begin{aligned}
\mathbb{P}\left\{\alpha B_{2}^{n} \not \subset K\right\} & \leq\left(\begin{array}{c}
2 m \\
n
\end{array}\right)\left(1-c^{\prime} e^{-4 \alpha^{2} n}\right)^{m-n} \\
& \leq\left(\frac{2 e m}{n}\right)^{n} \exp \left((m-n) \log \left(1-c^{\prime} e^{-4 \alpha^{2} n}\right)\right) \\
& \leq\left(\frac{2 e m}{n}\right)^{n} \exp \left(-c^{\prime}(m-n) e^{-4 \alpha^{2} n}\right) .
\end{aligned}
$$

Now, set $x=\frac{m}{n}$. Using the fact that there exists a constant $C$ such that $\sqrt{x}<\frac{c^{\prime}(x-1)}{\log x+2+\log 2}$ for all $x>C$, one can check that if $C n \leq m \leq n e^{\frac{n}{2}}$ and $\alpha=\frac{1}{2 \sqrt{2}} \sqrt{\frac{\log \frac{m}{n}}{n}}$, then

$$
\mathbb{P}\left\{\frac{1}{2 \sqrt{2}} \sqrt{\frac{\log \frac{m}{n}}{n}} B_{2}^{n} \not \subset K\right\} \leq e^{-n} .
$$

On the other hand, if $(1+\delta) n \leq m \leq C n$, since

$$
\begin{aligned}
\mathbb{P}\left\{P \in S^{n-1}:|\langle P, \theta\rangle| \leq \epsilon\right\} & =\frac{2(n-1) \omega_{n-1}}{n \omega_{n}} \int_{0}^{\epsilon}\left(1-x^{2}\right)^{\frac{n-3}{2}} d x \\
& \leq c \sqrt{n} \epsilon
\end{aligned}
$$


we have that if $c_{1}=c_{1}(\delta)$ is chosen small enough,

$$
\begin{aligned}
\mathbb{P}\left\{c_{1} \sqrt{\frac{\log \frac{m}{n}}{n}} B_{2}^{n} \nsubseteq K\right\} & \leq\left(\frac{2 e m}{n}\right)^{n}\left(c c_{1} \sqrt{\log \frac{m}{n}}\right)^{m-n} \\
& \leq(2 e C)^{n}\left(c c_{1}\right)^{m-n}(\log C)^{\frac{C n}{2}} \\
& \leq\left(2 e C\left(c c_{1}\right)^{\delta}(\log C)^{\frac{C}{2}}\right)^{n} \\
& \leq e^{-n}
\end{aligned}
$$

Hence

$$
\mathbb{P}\left\{\min \left\{c_{1}(\delta), \frac{1}{2 \sqrt{2}}\right\} \sqrt{\frac{\log \frac{m}{n}}{n}} B_{2}^{n} \nsubseteq K\right\} \leq e^{-n},
$$

and this completes the proof.

Now let us give an upper bound for $\frac{1}{|K|} \int_{K}|x|^{2} d x$. It is stated in the next theorem, whose proof follows the ideas in [6].

Theorem 3.1. There exist absolute constants $C$ and $C_{1}$ such that if $\left\{P_{i}\right\}_{i=1}^{m}$ are independent random vectors on $S^{n-1}, m>n$, and $K=\operatorname{conv}\left\{ \pm P_{1}, \ldots, \pm P_{m}\right\}$, then

$$
\mathbb{P}\left\{\frac{1}{|K|} \int_{K}|x|^{2} d x \leq C \frac{\log \frac{2 m}{n}}{n}\right\} \geq 1-2 e^{-C_{1} n \log \frac{m}{n}} .
$$

Proof. By Bernstein's inequality, if $\left\{P_{i}\right\}_{i=1}^{n}$ are independent random vectors on the sphere and if $\theta$ is a fixed point on the sphere, then, for all $\epsilon>0$,

$$
\mathbb{P}\left\{\left|\sum_{i=1}^{n}\left\langle P_{i}, \theta\right\rangle\right|>\epsilon n\right\} \leq 2 e^{-\frac{\epsilon^{2} n^{2}}{8 A^{2}}}
$$

Now let $\mathcal{N}$ be a $\frac{1}{2}$-net on the sphere such that $|\mathcal{N}| \leq 5^{n}$. Then

$$
\mathbb{P}\left\{\left|\sum_{i=1}^{n}\left\langle P_{i}, \theta\right\rangle\right|>\epsilon n \text { for some } \theta \in \mathcal{N}\right\} \leq 2 e^{-\frac{\epsilon^{2} n^{2}}{8 A^{2}}+n \log 5},
$$

and hence,

$$
\mathbb{P}\left\{\left|\sum_{i=1}^{n}\left\langle P_{i}, \theta\right\rangle\right| \leq \epsilon n \text { for every } \theta \in \mathcal{N}\right\} \geq 1-2 e^{-\frac{\epsilon^{2} n^{2}}{8 A^{2}}+n \log 5} .
$$

Every $\theta \in S^{n-1}$ can be written in the form $\theta=\sum_{j=1}^{\infty} \delta_{j} \theta_{j}$, with $\theta_{j} \in \mathcal{N}$ and $0 \leq \delta_{j} \leq\left(\frac{1}{2}\right)^{j-1}$; so, if for every $\theta \in \mathcal{N}$ it is true that $\left|\sum_{i=1}^{n}\left\langle P_{i}, \theta\right\rangle\right| \leq \epsilon n$, then for every $\theta \in S^{n-1}$

$$
\left|\left\langle\sum_{i=1}^{n} P_{i}, \theta\right\rangle\right|=\left|\left\langle\sum_{i=1}^{n} P_{i}, \sum_{j=1}^{\infty} \delta_{j} \theta_{j}\right\rangle\right| \leq \sum_{j=1}^{\infty} \delta_{j}\left|\left\langle\sum_{i=1}^{n} P_{i}, \theta_{j}\right\rangle\right| \leq 2 \epsilon n .
$$

Hence

$$
\mathbb{P}\left\{\left|\sum_{i=1}^{n} P_{i}\right| \leq 2 \epsilon n\right\}=\mathbb{P}\left\{\max _{\theta \in S^{n-1}}\left\langle\sum_{i=1}^{n} P_{i}, \theta\right\rangle \leq 2 \epsilon n\right\} \geq 1-2 e^{-\frac{\epsilon^{2} n^{2}}{8 A^{2}}+n \log 5}
$$


Now, since $\sum_{i \neq j}\left\langle P_{i}, P_{j}\right\rangle=\left|\sum_{i=1}^{n} P_{i}\right|^{2}-\sum_{i=1}^{n}\left|P_{i}\right|^{2}=\left|\sum_{i=1}^{n} P_{i}\right|^{2}-n$, this implies that if $\epsilon>\epsilon_{0}>32 A^{2} \log 5$, then

$$
\mathbb{P}\left\{\sum_{i \neq j}\left\langle P_{i}, P_{j}\right\rangle \leq \epsilon n\right\} \geq 1-2 e^{-C \epsilon n} .
$$

Let $\mathcal{F}_{1}, \ldots, \mathcal{F}_{l}$ denote a complete list of the facets of $K$. With probability one, each facet is of the form $\mathcal{F}_{k}=\operatorname{conv}\left\{Q_{1}^{k}, \ldots, Q_{n}^{k}\right\}$ with $Q_{i}^{k} \in\left\{ \pm P_{1}, \ldots, \pm P_{m}\right\}$ and with $Q_{i}^{k} \neq \pm Q_{j}^{k}$. Since $P$ and $-P$ have the same distribution, then

$$
\begin{aligned}
\mathbb{P}\left\{\max _{k=1 \ldots l} \sum_{i \neq j}\left\langle Q_{i}^{k}, Q_{j}^{k}\right\rangle>\epsilon n \log \frac{2 m}{n}\right\} & \leq\left(\begin{array}{c}
2 m \\
n
\end{array}\right) 2 e^{-C \epsilon n \log \frac{2 m}{n}} \\
& \leq 2 e^{-C \epsilon n \log \frac{2 m}{n}+n \log \left(\frac{2 e m}{n}\right)}
\end{aligned}
$$

whenever $\epsilon \log \frac{2 m}{n}>\epsilon_{0}$, so choosing a constant $\epsilon$ big enough we have that

$$
\mathbb{P}\left\{\max _{k=1 \ldots l} \sum_{i \neq j}\left\langle Q_{i}^{k}, Q_{j}^{k}\right\rangle>C n \log \frac{2 m}{n}\right\} \leq 2 e^{-C_{1} n \log \frac{m}{n}} .
$$

For each facet $\mathcal{F}_{k}=\operatorname{conv}\left\{Q_{1}^{k}, \ldots, Q_{n}^{k}\right\}$, let $T$ be the following linear transformation:

$$
T=\left(\begin{array}{ccc}
Q_{1}^{k}(1) & \ldots & Q_{n}^{k}(1) \\
\vdots & & \vdots \\
Q_{1}^{k}(n) & \ldots & Q_{n}^{k}(n)
\end{array}\right)
$$

Then $\mathcal{F}_{k}=T\left(\Delta^{n-1}\right)$ and

$$
\frac{1}{\left|\mathcal{F}_{k}\right|} \int_{\mathcal{F}_{k}}|x|^{2} d x=\frac{1}{\left|\Delta^{n-1}\right|} \int_{\Delta^{n-1}}|T x|^{2} d x
$$

Since

$$
T x=\left(\begin{array}{c}
\sum_{i=1}^{n} Q_{i}^{k}(1) x_{i} \\
\vdots \\
\sum_{i=1}^{n} Q_{i}^{k}(n) x_{i}
\end{array}\right)
$$

then

so

$$
|T x|^{2}=\sum_{j=1}^{n}\left(\sum_{i=1}^{n} Q_{i}^{k}(j) x_{i}\right)^{2}=\sum_{j=1}^{n} \sum_{i_{1}, i_{2}=1}^{n} Q_{i_{1}}^{k}(j) Q_{i_{2}}^{k}(j) x_{i_{1}} x_{i_{2}},
$$

$$
\frac{1}{\left|\mathcal{F}_{k}\right|} \int_{\mathcal{F}_{k}}|x|^{2} d x=\frac{1}{\left|\Delta^{n-1}\right|} \sum_{j=1}^{n} \sum_{i_{1}, i_{2}=1}^{n} Q_{i_{1}}^{k}(j) Q_{i_{2}}^{k}(j) \int_{\Delta^{n-1}} x_{i_{1}} x_{i_{2}} d x
$$

From the identity

$$
\frac{1}{\left|\Delta^{n-1}\right|} \int_{\Delta^{n-1}} x_{i_{1}} x_{i_{2}} d x=\frac{1+\delta_{i_{1} i_{2}}}{n(n+1)}
$$

which is proved in [6], this quantity equals

$$
\frac{1}{n(n+1)} \sum_{j=1}^{n}\left(\sum_{i=1}^{n} 2 Q_{i}^{k}(j)^{2}+\sum_{i_{1} \neq i_{2}} Q_{i_{1}}^{k}(j) Q_{i_{2}}^{k}(j)\right)=\frac{2}{n+1}+\frac{1}{n(n+1)} \sum_{i_{1} \neq i_{2}}\left\langle Q_{i_{1}}^{k}, Q_{i_{2}}^{k}\right\rangle
$$


and there exist absolute constants $C$ and $C_{1}$ such that the maximum of this quantity over all facets is less than $C \frac{\log \frac{2 m}{n}}{n}$ with probability bigger than $1-2 e^{-C_{1} n \log \frac{m}{n}}$, so

$$
\sup _{i=1 \ldots l} \frac{1}{\left|\mathcal{F}_{i}\right|} \int_{\mathcal{F}_{i}}|y|^{2} d y \leq C \frac{\log \frac{2 m}{n}}{n}
$$

with probability bigger than $1-2 e^{-C_{1} n \log \frac{m}{n}}$, where $C$ and $C_{1}$ are absolute constants.

But, in the same way as is proved in [6] we have that

- $\frac{1}{|K|} \int_{K}|x|^{2} d x=\frac{1}{|K|} \sum_{i=1}^{l} \frac{d\left(0, \mathcal{F}_{i}\right)}{n+2} \int_{\mathcal{F}_{i}}|y|^{2} d y$

- $n|K|=\sum_{i=1}^{l} d\left(0, \mathcal{F}_{i}\right)\left|\mathcal{F}_{i}\right|$,

where $d\left(0, \mathcal{F}_{i}\right)$ denotes the distance between the origin and the affine subspace generated by the facet $\mathcal{F}_{i}$. Hence

$$
\frac{1}{|K|} \int_{K}|x|^{2} d x \leq \frac{n}{n+2} \sup _{i=1 \ldots l} \frac{1}{\left|\mathcal{F}_{i}\right|} \int_{\mathcal{F}_{i}}|y|^{2} d y \leq C \frac{\log \frac{2 m}{n}}{n}
$$

with probability greater than $1-2 e^{-C_{1} n \log \frac{m}{n}}$.

Lemma 3.1 and Theorem 3.1 imply that for every $\delta>0$ there exist absolute constants $c(\delta), c_{1}, c_{2}, C_{1}$, such that if $(1+\delta) n<m \leq n e^{\frac{n}{2}}$, then

$$
\mathbb{P}\left\{L_{K} \leq c\right\} \geq 1-2 e^{-C_{1} n \log \frac{m}{n}}-e^{-n}>1-c_{1} e^{-c_{2} n \min \left\{1, \log \frac{m}{n}\right\}} .
$$

Note that in the case $m>n e^{\frac{n}{2}}$ we have that for $n$ large enough

$$
\mathbb{P}\left\{\frac{1}{4} B_{2}^{n} \nsubseteq K\right\} \leq \mathbb{P}\left\{\frac{1}{4} B_{2}^{n} \nsubseteq \operatorname{conv}\left\{ \pm P_{1}, \ldots, \pm P_{\left[n \exp \left\{\frac{n}{2}\right\}\right]}\right\}\right\} \leq e^{-n}
$$

so, with probability greater than $1-e^{-n}$,

$$
n L_{K}^{2} \leq \frac{1}{|K|^{\frac{2}{n}}} \frac{1}{|K|} \int_{K}|x|^{2} d x \leq \frac{1}{\left|\frac{1}{4} B_{2}^{n}\right|^{\frac{2}{n}}} \leq c n
$$

and the proof of Theorem 1.1 is complete.

\section{ACKNOWLEDGEMENTS}

This paper was written while the author was in an early-stage researcher position of the research training network "Phenomena in High Dimensions" (MRTNCT-2004-511953) in Athens. The author would like to thank Professor Apostolos Giannopoulos for several helpful discussions as well as for his hospitality. The author also thanks the referee for many comments that improved the presentation of this paper.

\section{REFERENCES}

[1] Ball, K. Normed spaces with a weak Gordon-Lewis property. Springer Lecture Notes in Math. 1470, Springer, Berlin (1991) pp. 36-47. MR1126735 (93e:46013)

[2] Bourgain, J. On the distribution of polynomials on high dimensional convex sets, Springer Lecture Notes in Math. 1469, Springer, Berlin (1991), pp. 127-137. MR1122617 (92j:52007)

[3] Bourgain, J.; Lindenstrauss, J.; Milman, V. D. Minkowski sums and symmetrizations. GAFA Seminar 1986-87, Springer Lecture Notes in Math. 1317, Springer, Berlin (1988), pp. 44-66. MR.950975 (89g:46025)

[4] Gluskin, E.D. Extremal properties of orthogonal parallelepipeds and their applications to the geometry of Banach spaces. Math. USSR-Sb. 64 (1989), pp. 85-96. English translation. MR945901 (89j:46016) 
[5] Klartag, B. On convex perturbations with a bounded isotropic constant. Geom. Funct. Anal. 16 (2006), no. 6, pp. 1274-1290. MR.2276540 (2007i:52005)

[6] Klartag, B.; Kozma, G. On the hyperplane conjecture on random convex sets. (Preprint).

[7] König, H.; Meyer, M.; Pajor, A. The isotropy constants of the Schatten classes are bounded. Math. Ann. 312 (1998), no. 4, pp. 773-783. MR.1660231(99j:52003)

[8] Litvak, A.E.; Pajor, A.; Rudelson, M.; Tomczak-Jaegermann, N. Smallest singular value of random matrices and geometry of random polytopes. Adv. Math. 195 (2005), pp. 491-523. MR2146352(2006g:52009)

[9] Milman, V.; Pajor, A. Isotropic position and inertia ellipsoids and zonoids of the unit ball of a normed $n$-dimensional space, GAFA Seminar 1987-88, Springer Lecture Notes in Math. 1376, Springer, Berlin (1989), pp. 64-104. MR1008717 (90g:52003)

Institute of Mathematics, Universidad de Zaragoza, 50009 Zaragoza, Spain

E-mail address: daalonso@unizar.es 